\title{
Erratum
}

\section{Compact hyperkähler manifolds: basic results}

\section{Daniel Huybrechts}

Institut de Mathématiques, Université de Paris 7 - Denis Diderot, Case 7012, 2, place Jussieu, 75251 Paris Cedex 05, France (e-mail: huybrech@math.jussieu.fr)

Invent. math. 135, 63-113 (1999)

Oblatum 20-VIII-2001 \& 13-XI-2002

Published online: 17 February 2003 - (C) Springer-Verlag 2003

It was pointed out by D. Kaledin that the proof of Proposition 3.8 is wrong. Actually, the proposition itself cannot be true as we shall explain below. It was used to prove Corollary 3.10 and Theorem 3.11. The latter is the so called projectivity criterion for hyperkähler manifolds. Here we will give a correct proof of these two results. The main input is a recent theorem of J.-P. Demailly and M. Paun [3]. All the other results of the paper remain unaffected.

The projectivity criterion for hyperkähler manifolds as a consequence of the Demailly-Paun theorem

1. Let $X$ be a compact hyperkähler manifold of complex dimension $2 n$ and let $X \rightarrow \operatorname{Def}(X)$ be the universal deformation of $X$. For any cohomology class $\beta \in H^{4 p}(X, \mathbb{R})$ let $S_{\beta} \subset \operatorname{Def}(X)$ be the set of those $t \in \operatorname{Def}(X)$ for which $\beta$ is a cohomology class of type $(2 p, 2 p)$ on $X_{t}$. Then $S_{\beta}$ is a closed analytic subset of $\operatorname{Def}(X)$. Let $\mathrm{A} \subset H^{*}(X, \mathbb{Z})$ be the set of all integral classes $\beta \in H^{4 p}(X, \mathbb{Z}), p=1, \ldots, n$ such that $S_{\beta}$ is a proper subset of $\operatorname{Def}(X)$. A point $t \in \operatorname{Def}(X)$ is called very general if $t$ is in the complement of $\bigcup_{\beta \in \mathrm{A}} S_{\beta}$ and if $X_{t}$ does not admit any analytic subsets of odd dimension.

The set of very general points in $\operatorname{Def}(X)$ is dense. If $t \in \operatorname{Def}(X)$ is a very general point and $\beta \in H^{2 p, 2 p}\left(X_{t}, \mathbb{Z}\right)$ is an integral class of type $(2 p, 2 p)$ on $\mathcal{X}_{t}$, then $\beta$ is of type $(2 p, 2 p)$ on any small deformation of $\mathcal{X}_{t}$.

Here we use Fujiki's result [5, Prop. 5.11] and the fact that $\bigcup_{\beta \in \mathrm{A}} S_{\beta}$ is a countable union of proper closed analytic subsets. Fujiki's result says that 
for any hyperkähler metric on $X$ the general complex structure compatible with it does not admit any odd-dimensional analytic subset.

For classes $\beta$ which are of pure type $(2 p, 2 p)$ on any small deformation of $X$ the form of degree $2 n-2 p$ on $H^{2}(X, \mathbb{C})$ defined by $\alpha \mapsto \int_{X} \beta \alpha^{2(n-p)}$ can be expressed in terms of the Beauville-Bogomolov quadratic form $q_{X}$ on $H^{2}(X, \mathbb{C})$. More precisely we have:

If $\beta \in H^{4 p}(X, \mathbb{C})$ is of type $(2 p, 2 p)$ on all small deformations of $X$, then there exists a constant $c_{\beta}$ depending on $\beta$ such that for all $\alpha \in H^{2}(X, \mathbb{C})$ one has $\int_{X} \beta \alpha^{2(n-p)}=c_{\beta} q_{X}(\alpha)^{n-p}$.

This is Theorem 5.12 in [7], which is a generalization of a result of Fujiki and whose proof uses arguments of Bogomolov (cf. [6, 1.11]).

2. We call the compact hyperkähler manifold $X$ itself very general if the point $0 \in \operatorname{Def}(X)$ corresponding to it is a very general point. As a consequence of the above one obtains:

If $X$ is a very general compact hyperkähler manifold and $Y \subset X$ is an irreducible analytic subset then its codimension is even, say $2 p$, and the cohomology class $[Y] \in H^{2 p, 2 p}(X, \mathbb{Z})$ is of type $(2 p, 2 p)$ on all small deformations of $X$. In particular, there exists a constant $c_{[Y]}$ such that $\int_{Y} \alpha^{2(n-p)}=c_{[Y]} q_{X}(\alpha)^{n-p}$ for all $\alpha \in H^{2}(X, \mathbb{C})$.

We next quote the result of Demailly and Paun:

Theorem 1 [3] Let $X$ be a compact Kähler manifold. Then the Kähler cone $\mathcal{K}_{X}$ of $X$ is a connected component of the set $\mathcal{P}_{X}$ of all classes $\alpha \in H^{1,1}(X, \mathbb{R})$ such that $\int_{Y} \alpha^{d}>0$ for any irreducible analytic subset $Y \subset X$ of dimension $d$.

Combining this with the above one obtains a description of the Kähler cone of a very general hyperkähler manifold. Recall that the positive cone $\mathcal{C}_{X} \subset H^{1,1}(X, \mathbb{R})$ is the connected component of the open subset $\{\alpha \in$ $\left.H^{1,1}(X, \mathbb{R}) \mid q_{X}(\alpha)>0\right\}$ that contains the Kähler cone $\mathcal{K}_{X}$.

Corollary 1 Let $X$ be a very general compact hyperkähler manifold. Then $\mathcal{K}_{X}=\mathcal{C}_{X}$. Moreover, the interior of the cone of pseudo-effective classes coincides with $\mathcal{K}_{X}=\mathcal{C}_{X}$.

Proof. One first shows that $\mathcal{C}_{X} \subset \mathcal{P}_{X}$. Since $\mathcal{K}_{X} \subset \mathcal{P}_{X}$ and $\mathcal{C}_{X}$ is connected, it suffices to show that for any $\alpha \in \mathcal{C}_{X}$ and any irreducible analytic subset $Y \subset X$ of codimension $2 p$ one has $\int_{Y} \alpha^{2(n-p)} \neq 0$. Since $\int_{Y} \alpha^{2(n-p)}=c_{[Y]} q_{X}(\alpha)^{n-p}$ and $q_{X}(\alpha)>0$, this follows from $c_{[Y]} \neq 0$. The latter can be obtained from the same equation applied to a Kähler class. Thus, $\mathcal{C}_{X} \subset \mathcal{P}_{X}$. Since $\mathcal{C}_{X}$ is connected and contains $\mathcal{K}_{X}$, the DemaillyPaun theorem shows that $\mathcal{C}_{X}=\mathcal{K}_{X}$. 
Clearly, every class in $\mathcal{K}_{X}=\mathcal{C}_{X}$ is in the interior of the cone of pseudo-effective classes, which consists of the cone of classes that can be represented by closed positive $(1,1)$-currents bounded from below by a Kähler form. Conversely, if $\alpha$ can be represented by a closed strictly positive $(1,1)$-current then $q_{X}(\alpha, \omega)=c \int(\sigma \bar{\sigma})^{n-1} \alpha \omega>0$ for any Kähler class $\omega \in \mathcal{K}_{X}=\mathcal{C}_{X}$. Here, $c$ is a certain positive scalar and $\sigma$ is a nondegenerate holomorphic two-form. Hence, $\alpha \in \mathcal{C}_{X}$.

3. Since the set of very general $t \in \operatorname{Def}(X)$ is dense, any class $\alpha \in \mathcal{C}_{X} \subset$ $H^{2}(X, \mathbb{R})$ can be approximated by a sequence $\alpha_{t_{i}} \in H^{2}(X, \mathbb{R})$ such that $t_{i}$ is a sequence of very general points converging to $0 \in \operatorname{Def}(X)$ and $\alpha_{t_{i}}$ is a Kähler class on $\mathcal{X}_{t_{i}}$. This is enough to conclude:

Proposition 1 Let $X$ be a compact hyperkähler manifold and let $\alpha \in \mathcal{C}_{X}$ Then $\alpha$ is in the interior of the cone of pseudo-effective classes, i.e. $\alpha$ can be represented by a closed positive $(1,1)$-current which can be bounded from below by a (small) Kähler form.

Proof. Here one can copy the argument of Demailly [4, Prop. 6.1] that shows that the cone of pseudo-effective classes is closed. Fix Kähler classes $\omega_{t}$ on $\mathcal{X}_{t}$ depending continously on $t \in \operatorname{Def}(X)$. Then the mass of $\alpha_{t_{i}}$, which is $\int_{X} \omega_{t_{i}}^{2 n-1} \alpha_{t_{i}}$, converges to $\int_{X} \omega_{0}^{2 n-1} \alpha$. Hence the sequence of forms $\left(\alpha_{t_{i}}\right)$ is weakly bounded and thus weakly compact. In particular, it contains a weakly convergent subsequence. As the $\alpha_{t_{i}}$ are closed positive $(1,1)$-forms on $\mathcal{X}_{t_{i}}$, the limit current is closed and positive of bidegree $(1,1)$ on $X$. As $\mathcal{C}_{X}$ is open, the class $\alpha$ must be in the interior of the cone of all pseudo-effective classes.

As a consequence we obtain the projectivity criterion for compact hyperkähler manifolds which was stated as Theorem 3.11 in [6], but the proof of which was seriously flawed as it used the wrong Proposition 3.8.

Theorem 2 Let $X$ be a compact hyperkähler manifold. Then $X$ is projective if and only if there exists a line bundle $L$ on $X$ with $q_{X}\left(c_{1}(L)\right)>0$.

Proof. If $X$ is projective, then there exists an ample line bundle $L$. As $c_{1}(L)$ is then a Kähler class and $\mathcal{K}_{X} \subset \mathcal{C}_{X}$, this yields $q_{X}\left(c_{1}(L)\right)>0$. Conversely, let us assume that there exists a line bundle $L$ with $q_{X}\left(c_{1}(L)\right)>0$. This is equivalent to the existence of a line bundle $L$ with $c_{1}(L) \in \mathcal{C}_{X}$. By the previous proposition $c_{1}(L)$ is in the interior of the cone of pseudoeffective classes. Thus, $c_{1}(L)$ can be represented by a closed positive $(1,1)$ current which is bounded from below by a Kähler form. Applying results of Bonavero [1] and Ji-Shiffman [9] one obtains that $X$ is Moishezon and hence projective.

4. Let me indicate why Proposition 3.8 in [6] has to be false. One way to see this is to use the fact that from dimension four on, the birational Kähler 
cone can really be different from the Kähler cone itself (something that does not happen for K3 surfaces). In this case one just picks a class that is positive on the Kähler cone, but not on the entire birational Kähler cone. If the original Proposition 3.8 were true, this class would be representable by a closed positive current. But considered on any other birational compact hyperkähler manifold it would also be representable by a closed positive current, which is in contradiction with the fact that there is at least on birational compact hyperkähler manifold where the class is not positive on the Kähler cone. In this argument we use that the quadratic form $q_{X}$ is compatible with birational correspondences.

5. Some of the results of the paper have been strengthened in the meantime. In [8] we prove Theorem 4.6. without assuming the projectivity of the varieties. A better description of the closure of the Kähler cone than the one given in Theorem 7.1 has also been found: A class is in the closure of the Kähler cone if and only if it is non-negative on all rational curves (Proposition 3.1 in [8]). In fact, Boucksom [2] extended this result to a description of the open Kähler cone.

Acknowledgements. First of all I wish to thank D. Kaledin for pointing out the blunder in the proof of Prop. 3.8 and for believing in the projectivity criterion and its consequences all the time. He was always very patient to check various arguments over the last two years. I am certainly most grateful to J.-P. Demailly and M. Paun for their beautiful theorem. Thanks to J.-P. Demailly for comments on the new proof.

\section{References}

1. Bonavero, L.: Inégalités de Morse holomorphes singulières. C. R. Acad. Sci., Paris, Sér. I, Math. 317, 1163-1166 (1993)

2. Boucksom, S.: Le cône kählérien d'une variéé hyperkählérienne. C. R. Acad. Sci., Paris, Sér. I, Math. 333, 935-938 (2001)

3. Demailly, J.-P., Paun, M.: Numerical characterization of the Kähler cone of a compact Kähler manifold. math.AG/0105176. To appear in Ann. Math.

4. Demailly, J.-P.: Regularization of closed positive currents and intersection theory. J. Algebr. Geom. 1, 361-409 (1992)

5. Fujiki, A.: On the de Rham Cohomology Group of a Compact Kähler Symplectic Manifold. Adv. Stud. Pure Math. 10, 105-165 (1987)

6. Huybrechts, D.: Compact Hyperkähler Manifolds: Basic Results. Invent. math. 135, 63-113 (1999)

7. Huybrechts, D.: Compact hyperkähler manifolds. Habilitation (1997). http://www.mi.uni-koeln.de/ huybrech/artikel.htm/HKhabmod.ps

8. Huybrechts, D.: The Kähler cone of a compact hyperkähler manifold. math.AG/9909109. To appear in Math. Ann.

9. Ji, S., Shiffman, B.: Properties of compact complex manifolds carrying closed positive currents. J. Geom. Anal. 3, 37-61 (1993) 